EPJ Web of Conferences 36, 00014 (2012)

DOI: $10.1051 /$ epjconf/20123600014

(C) Owned by the authors, published by EDP Sciences, 2012

\title{
Resonances from AMPT
}

\author{
Che Ming $\mathrm{Ko}^{1, \mathrm{a}}$ \\ ${ }^{1}$ Cyclotron Institute and Department of Physics and Astronomy, College Station, Texas 77843-3366, \\ USA
}

\begin{abstract}
A multiphase transport (AMPT) model that includes both initial partonic and final hadron rescattering effects in relativistic heavy ion collisions is briefly reviewed. Particular attention is given to the scattering processes in the hadronic phase in order to address resonances production in these collisions. As an example, phi meson production is discussed in detail through both its dikaon and dilepton decay channels. Results from the AMPT model for heavy ion collisions at both SPS and RHIC are compared to the experimental data to extract information on the production mechanism and in-medium properties of the phi meson.
\end{abstract}

\section{Introduction}

The study of resonances in relativistic heavy ion collisions offers the possibility to investigate their in-medium properties as well as those of the hot and dense matter produced in these collisions. For example, from the study of dileptons from the rho meson decay, it has been found that the rho meson mass remains essentially unchanged in hot dense matter, although its width becomes significantly widened $[1,2]$. Also, the yield of $\Lambda(1520)$ relative that of $\Lambda(1115)$ [3] has been found to be significantly less than that predicted by the thermal model [4], which has been very successful in describing the relative yields of stable hadrons. In this model, the abundance of produced particles is fixed at the chemical freeze out temperature that is close to the critical temperature at which the initially produced quarkgluon plasma undergoes a phase transition to the hadronic matter. The measured $\Lambda(1520) / \Lambda(1115)$ ratio is, however, similar to that calculated in the UrQMD model [5], thus indicating the importance of hadronic scattering on resonance production in relativistic heavy ion collisions. Here, I briefly review the the multiphase AMPT model [6] and describe, in particular, the hadronic scattering processes that are included in this model. I then use the phi meson as an example to illustrate the importance of hadronic scattering on its final yield and transverse momentum spectrum [7]. I also discuss the medium effects on phi meson production. From comparing the results from AMPT on phi mesons with experimental data from heavy ion collisions at SPS and RHIC, I further discuss what one can learn from relativistic heavy ion collisions about the properties of phi mesons in medium.

\section{The AMPT model}

The AMPT model is a hybrid model that uses minijet partons from hard processes and strings from soft processes in the heavy ion jet interaction generator (HIJING) model [8] as initial conditions for modeling heavy-ion collisions at ultra-relativistic energies. In the default version [9], time evolution of the minijet partons, which are largely gluons, is described by Zhang's parton cascade (ZPC) model [10].

\footnotetext{
a Supported by the U.S. National Science Foundation under Grant No. PHY-1068572, the US Department of Energy under Contract No. DE-FG02-10ER41682, and the Welch Foundation under Grant No. A-1358. e-mail: ko@comp.tamu.edu
}

This is an Open Access article distributed under the terms of the Creative Commons Attribution License 2.0, which permits unrestricted use, distribution, and reproduction in any medium, provided the original work is properly cited. 
At present, this model includes only parton-parton elastic scatterings with an in-medium cross section given by the perturbative QCD with the gluon screening mass taking as a parameter for fixing its magnitude and angular distribution. After minijet partons stop interacting, they are combined with their parent strings, as in the HIJING model with jet quenching, to fragment into hadrons using the Lund string fragmentation model as implemented in the PYTHIA program [11]. The final-state hadronic scatterings are further modeled by a relativistic transport (ART) model [12]. In the string melting version of the AMPT model [13], hadrons that would have been produced from the HIJING model through the fragmentation of strings with attached minijets are converted to their valence quarks and/or antiquarks to take into account the effect due to the larger initial energy density in ultra-relativistic heavy ion collisions than the critical energy density for the hadronic matter to quark-gluon plasma transition. Interactions among these partons are again described by the ZPC parton cascade model. Because inelastic scatterings are not included in the current version of the ZPC model, only quarks and antiquarks from melted strings are present in the partonic matter. The transition from the partonic matter to the hadronic matter in the AMPT with string melting is achieved using a coordinate-space quark coalescence model, i.e., two nearest quark and antiquark are combined into mesons and three nearest quarks or antiquarks are combined into baryons or anti-baryons that are closest to the invariant masses of these parton combinations. The final-state scatterings of produced hadrons from quark coalescence in this extended AMPT model are again described by the ART model.

\section{The ART model}

The ART model is a relativistic transport model for describing the time evolution of the hadronic matter produced in relativistic heavy ion collisions. It includes the following baryons with all possible charge states: $N, \Delta(1232), N^{*}(1440), N^{*}(1535), \Lambda, \Sigma$, and mesons: $\pi, \rho, \omega, \eta, K$. The one used in AMPT further includes the $K^{*}$ resonance, multistrange baryons $\Xi$ and $\Omega$, and anti-baryons [14]. Both elastic and inelastic collisions among most of these particles are included as best as one can by using the experimental data from hadron-hadron collisions.

For baryon-baryon scatterings, the ART model includes following inelastic channels: $N N \leftrightarrow$ $N\left(\Delta N^{*}\right), N N \leftrightarrow \Delta\left(\Delta N^{*}(1440)\right), N N \leftrightarrow N N(\pi \rho \omega),(N \Delta) \Delta \leftrightarrow N N^{*}$, and $\Delta N^{*}(1440) \leftrightarrow N N^{*}(1535)$. In the above, $N^{*}$ denotes either $N^{*}(1440)$ or $N^{*}(1535)$, and the symbol $\left(\Delta N^{*}\right)$ denotes a $\Delta$ or an $N^{*}$, Also included are reaction channels relevant for kaon production, i.e., $\left(N \Delta N^{*}\right)\left(N \Delta N^{*}\right) \rightarrow(N \Delta)(\Lambda \Sigma) K$.

For meson-baryon scatterings, the ART model includes following reaction channels for the formation and decay of resonances: $\pi N \leftrightarrow\left(\Delta N^{*}(1440) N^{*}(1535)\right)$, and $\eta N \leftrightarrow N^{*}(1535)$. There are also elastic scatterings such as $(\pi \rho)\left(N \Delta N^{*}\right) \rightarrow(\pi \rho)\left(N \Delta N^{*}\right)$ with cross sections evaluated by including heavier baryon resonances with masses up to $2.0 \mathrm{GeV} / c^{2}$ as intermediate states using the Breit-Wigner form but neglecting interferences between the amplitudes from different resonances. The ART model further includes inelastic reaction channels such as $\pi N \leftrightarrow(\pi \rho \eta) \Delta$ and kaon production channels such as $(\pi \rho \omega \eta)\left(N \Delta N^{*}\right) \leftrightarrow K(\Lambda \Sigma)$. Kaon elastic scatterings with nucleons and baryon resonances are included with a constant cross section of $10 \mathrm{mb}$. Antikaon elastic scatterings with nucleons and inelastic channels, such as $\bar{K}\left(N \Delta N^{*}\right) \leftrightarrow \pi(\Lambda \Sigma)$, are included [15] using parameterized experimental data [16]. Also included are kaon production channels involving three-body final states, $(\pi \rho \omega)\left(N \Delta N^{*}\right) \rightarrow K \bar{K} N$ [15]. Because of the difficulty associated with the three-body kinematics, the inverse kaon annihilation reactions of above channels are neglected.

For meson-meson interactions, the ART model includes both elastic and inelastic $\pi \pi$ interactions, with the elastic cross section consisting of $\rho$ meson formation and the remaining part treated as elastic scattering. Kaon production from inelastic scatterings of light mesons is included via the reactions $(\pi \eta)(\pi \eta) \leftrightarrow K \bar{K}$ and $(\rho \omega)(\rho \omega) \leftrightarrow K \bar{K}$. Kaon or antikaon elastic scatterings with mesons in the SU(2) multiplets except the pion are included using a constant cross section of $10 \mathrm{mb}$, while the kaon-pion elastic scattering is modeled through the $K^{*}$ resonance. In addition to its formation from $\pi K$ scattering and its decay, elastic scatterings of $K^{*}$ with $(\rho \omega \eta)$ are included using same constant cross section of $10 \mathrm{mb}$ as those for kaons. Inelastic reaction channels of $(\pi \eta)(\rho \omega) \leftrightarrow K^{*} \bar{K}$ or $\bar{K}^{*} K$ and $\pi K \leftrightarrow K^{*}(\rho \omega)$ are also included [17]. Furthermore, inelastic meson-meson collisions such as $\pi \pi \leftrightarrow \rho \rho$ are included as well as the elastic scatterings between $\pi$ and $(\rho \omega \eta)$ with cross sections taken to be $20 \mathrm{mb}$. Inelastic 
scatterings of $\eta$ meson with other mesons are, however, taken to be $5 \mathrm{mb}$ [18], which is roughly in line with theoretical predictions based on the Hidden Local Symmetry Lagrangian [19].

The ART model also includes antibaryon resonances such as $\Delta$ and $\bar{N}^{*}$ with their formations, decays, and scatterings analogous to those of baryon resonances. It further includes $\left(N \Delta N^{*}\right)\left(\bar{N} \Delta \bar{N}^{*}\right)$ annihilation and also the inverse reactions of baryon-antibaryon pair production from mesons [20]. The latter is treated by assuming that the final state of three pions is equivalent to a $\pi \rho$ state; the four-pion final state is equivalent to $\rho \rho$ and $\pi \omega$ with equal probabilities; the five-pion state is equivalent to $\rho \omega$; and the six-pion state is equivalent to $\omega \omega$. The cross sections for baryon-antibaryon pair production from two mesons are then obtained from detailed balance relations.

For the production of multistrange baryons such as $\Xi$ and $\Omega$, it is included through the strangenessexchange reactions such as $\bar{K}(\Lambda \Sigma) \leftrightarrow \pi \Xi, \bar{K} \Xi \leftrightarrow \pi \Omega$. Interactions of these strange baryons as well as their anti-particles with mesons are included but not their annihilations by baryons.

The ART model also includes $\phi$ meson formation from and decay to kaon-antikaon pair with the formation cross section given by the standard Breit-Wigner form. Inelastic scatterings of the phi meson include baryon-baryon channels, $\left(N \Delta N^{*}\right)\left(N \Delta N^{*}\right) \rightarrow \phi N N$, and meson-baryon channels, $(\pi \rho)\left(N \Delta N^{*}\right) \leftrightarrow \phi\left(N \Delta N^{*}\right)$ with the cross sections for the forward-going reactions taken from the oneboson-exchange model [21]. The meson-baryon channels also include $K(\Lambda \Sigma) \leftrightarrow \phi N$ with cross section taken from a kaon-exchange model [22]. Phi meson scatterings with mesons such as $\pi, \rho, K$ and $\phi$, i.e., $\phi(\pi \rho \omega) \leftrightarrow\left(K K^{*}\right)\left(\bar{K} \bar{K}^{*}\right)$, and $\phi\left(K K^{*}\right) \leftrightarrow(\pi \rho \omega)\left(K K^{*}\right)$ are included with cross sections determined from the partial collisional widths given in Ref. [23]. The cross section for the elastic scattering of the phi meson with a nucleon is set to $8 \mathrm{mb}$ while that with a meson is set to $5 \mathrm{mb}$ except that the $\phi K$ elastic cross section is taken to be $2 \mathrm{mb}[7]$.

\section{4 phi meson production in heavy ion collisions}

As an example for resonance production in AMPT, we discuss in this Section phi meson production from heavy ion collisions at SPS and RHIC as reported in Ref. [7] based on the default AMPT model. Phi meson is an interesting resonance as it can decay into either dikaon or dilepton as in the case of the rho meson. This makes it possible to study its properties in the produced hot dense matter and to use it also as a probe to the latter's properties.

\subsection{SPS}

\subsection{1 rapidity distribution}

The rapidity distribution of phi mesons reconstructed from $K^{+} K^{-}$and $\mu^{+} \mu^{-}$channels in central $\mathrm{Pb}+\mathrm{Pb}$ collision at $158 \mathrm{~A} \mathrm{GeV}$ is shown in Fig. 1. In absence of any medium effects, the results for phi mesons from the the $\mathrm{K}^{+} \mathrm{K}^{-}$channel (thin solid curve) are in good agreement with the NA49 experimental data [24] (solid circles). With free-space decay width of phi meson, the rapidity distribution of all phi mesons from the $K^{+} K^{-}$, neglecting their scattering, would be identical to that reconstructed from the $\mu^{+} \mu^{-}$channel (thin dashed curve). Due to rescattering or absorption of the kaon pairs, $\sim 30 \%$ of all $\phi$ mesons are lost in the reconstruction, of which about $40 \%$ of the decaying kaons have at least one (anti)kaon that suffer elastic scattering. The maximum depletion of phi mesons from the $K^{+} K^{-}$channel occurs at midrapidity where kaon-antikaon pairs undergo appreciable scattering in the dense hadronic medium. Around the rapidity $y=0$, phi meson from dimuon channel is about a factor 1.7 larger than from the kaon-antikaon channel, which is smaller than the factor of 2-4 enhancement observed in the NA50 data [25].

\subsection{2 transverse mass spectrum}

In Fig. 2, we show the transverse mass spectra at midrapidity for phi meson from kaon-antikaon (solid squares) and dimuon (open squares) channel without medium effects. It is seen that at low $m_{T}$ the 


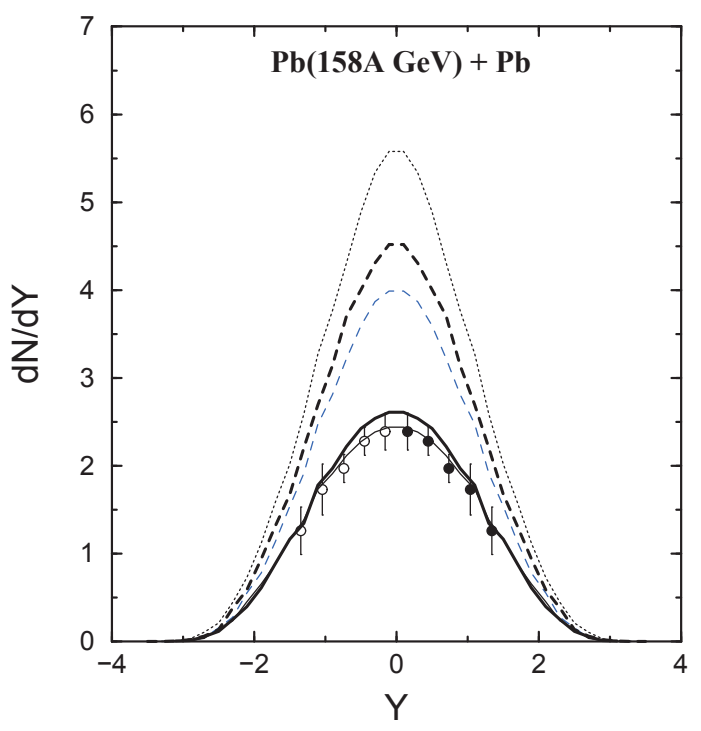

Fig. 1. Rapidity distribution of phi meson reconstructed from $K^{+} K^{-}$pairs (solid curves) and from $\mu^{+} \mu^{-}$channel (dashed curves) for $\mathrm{Pb}+\mathrm{Pb}$ collisions at $158 \mathrm{~A} \mathrm{GeV}$ at an impact parameter of $b \leq 3.5 \mathrm{fm}$ in the AMPT model. The results are for without (thin curves) and with (thick curves) in-medium mass modifications. The dotted curve corresponds to phi mesons from the dimuon channel with in-medium masses and with the phi meson number from HIJING increased by a factor of two. The solid circles are the NA49 experimental data [24] from the $K^{+} K^{-}$ channel.

phi meson from $\mathrm{K}^{+} \mathrm{K}^{-}$channel is suppressed due to rescattering. Since the transverse momentum of a particle increases due to increasing number of scattering and due to pressure build-up inside the system, the decayed kaons at the early stages, which are predominantly scattered, thus have low transverse momenta. The transverse mass spectra can be approximately fitted by $\exp \left(-m_{T} / T\right)$. The inverse slope parameter $T$ reported by NA50 [25] from the $\mu^{+} \mu^{-}$channel for $1.7<m_{T}<3.2 \mathrm{GeV} / \mathrm{c}^{2}$ at midrapidity is $T=227 \pm 10 \mathrm{MeV}$, which agrees well with the AMPT model prediction of $T=228 \mathrm{MeV}$. In contrast, the slope parameter extracted by NA49 [24] from the $K^{+} K^{-}$channel for $1<m_{T}<2.2$ $\mathrm{GeV} / \mathrm{c}^{2}$ is $T=305 \pm 15 \mathrm{MeV}$. The depletion of reconstructed $\phi$ at low $m_{T}$ in the AMPT model leads only to a slighter higher slope of $T=267 \mathrm{MeV}$, and thus is much smaller than the NA49 data. The ratio of the yields at midrapidity, $R\left(m_{T}\right)=N_{K^{+} K^{-}}\left(\Gamma_{\phi} / \Gamma_{\phi \rightarrow K \bar{K}}\right) / N_{\mu^{+} \mu^{-}}\left(\Gamma_{\phi} / \Gamma_{\phi \rightarrow \mu \mu}\right)$, corrected by their respective branching ratios, is shown in the inset of Fig. 2 . The maximum suppression of $\sim 40 \%$ at low $m_{T}$ in the kaon channel as observed in the AMPT model (squares) is smaller than the suppression factor of $60 \%$ found in the RQMD calculation [26]. This can be traced back to enhanced phi meson production from large collisional scattering among the mesons leading to a peak in the $\phi$ yield at the early stage. The dimuons from these phi mesons escape the fireball freely while the $K^{+} K^{-}$are rescattered in the dense hadronic medium and do not contribute to the reconstruction of phi mesons.

\subsubsection{In-medium effect}

Studies based on various approaches, such as the relativistic mean-field theory [27] and the chiral effective Lagrangian [28], have led to a general consensus that $K^{+}$feels a rather weak repulsive potential while the $K^{-}$feels a relatively stronger attractive potential. Including such potentials in the transport model has led to a successful description of the kaon yield and spectrum measured at both SIS energies (1-2A GeV) [29-31] and the AGS energy ( 11A GeV) [32]. Adopting the kaon and antikaon 


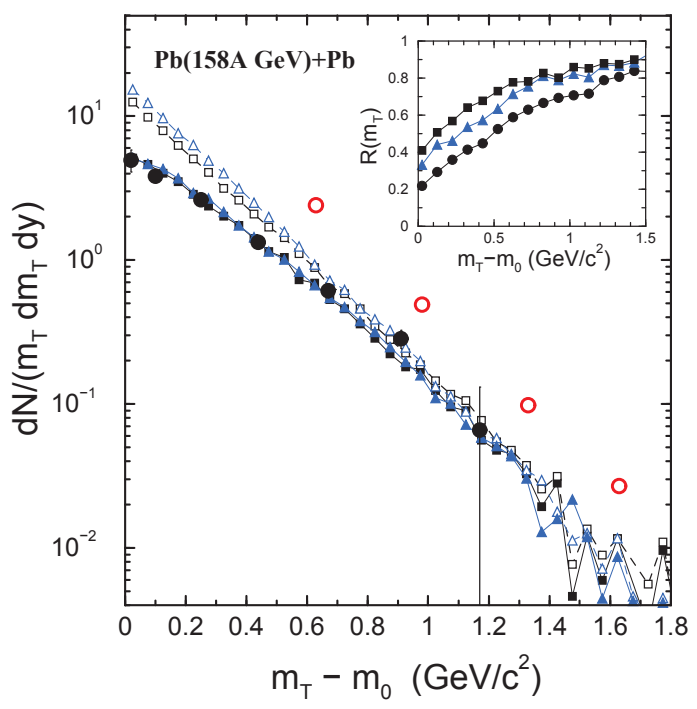

Fig. 2. Transverse mass spectra for midrapidity $(|y|<1)$ phi mesons reconstructed from $K^{+} K^{-}$pairs (solid symbols) and from $\mu^{+} \mu^{-}$channel (open symbols) for $\mathrm{Pb}+\mathrm{Pb}$ collisions at $158 \mathrm{~A} \mathrm{GeV}$ at an impact parameter of $b \leq 3.5$ $\mathrm{fm}$ in the AMPT model. The results are for without (squares) and with (triangles) in-medium mass modifications. The solid circles are the NA49 data [24] for $\phi \rightarrow K^{+} K^{-}$decay, and the open circles are the NA50 data [39] for $\phi \rightarrow \mu^{+} \mu^{-}$. In the inset is shown as a function of $m_{T}$ the ratio $R\left(m_{T}\right)$ for phi mesons decaying to kaon-antikaon pairs that are not scattered to those determined from the dimuon channel. The results are for without (squares) and with (triangles) in-medium mass modification, and with a further increase of phi meson number by two in the HIJING model (circles).

energies from chiral effective Lagrangian, i.e., $\omega_{K, \bar{K}}=\left[m_{K}^{2}+\mathbf{k}^{2}-a_{K} \rho_{S}+\left(b_{K} \rho\right)^{2}\right]^{1 / 2} \pm b_{K} \rho$, where $b_{K}=3 f_{\pi}^{2} / 8 \approx 0.333 \mathrm{GeV} \mathrm{fm}{ }^{3}, a_{K} \approx 0.22 \mathrm{GeV}^{2} \mathrm{fm}^{3}$ for kaons, and $a_{\bar{K}} \approx 0.45 \mathrm{GeV}^{2} \mathrm{fm}^{3}$ for antikaons. The $K^{+}$potential, defined by $U_{K}=\omega_{K}-\sqrt{m_{K}^{2}+\mathbf{k}^{2}}$ and is then $\sim+20 \mathrm{MeV}$ at normal nuclear density and is consistent with that determined from the impulsive approximation using the empirical kaon-nucleon scattering length [33]. The $K^{-}$potential at normal nuclear density is about $-110 \mathrm{MeV}$ and is somewhat less than that extracted from the kaonic atoms [34]. The present set of parameters was found to reproduce well the $K^{+}$and $K^{-}$kinetic energy spectra and the ratio of their yields for heavy ion collisions at the SIS energy [35].

For the in-medium phi meson mass, the QCD sum rule studies show that it decreases slightly in hot dense matter [36,37]. Using the result from Ref. [36], we have $m_{\phi}^{*} / m_{\phi} \approx 1-0.0255 \rho / \rho_{0}$, where $\rho_{0}$ is the normal nuclear matter density. Although the phi meson mass decreases in the medium, the larger reduction of the overall kaon-antikaon mass with increasing density results in an increase of phi meson width $\Gamma_{\phi \rightarrow K \bar{K}}^{*}$ in the medium. At density $\rho=2 \rho_{0}$, the phi meson in-medium width is about 45 $\mathrm{MeV}$. This result is in qualitative agreement with that from the QCD sum rule study that takes into account both the phi meson in-medium mass and width [38].

The rapidity distribution of $\phi$ from kaon pairs that have not suffered any collision is shown in Fig. 1 with in-medium masses (thick solid curve), which is only slightly above that without medium effects and thus lie within the error bars of the NA49 data. Since free-space branching ratio is used to determine the phi meson abundance from the kaon channel, it is evident that large in-medium width $\Gamma_{\phi \rightarrow K \bar{K}}^{*}$ leads to appreciable production and simultaneous decay of phi meson, resulting in $d N / d y \approx$ 7.1 at $y \approx 0$ for all these kaon pairs including those having been rescattered. In contrast, since the branching ratio for $\phi \rightarrow \mu^{+} \mu^{-}$is largely unaltered in the medium, the $d N / d y$ of the reconstructed phi mesons at $y \approx 0$ is 4.5 and thus about 1.9 times larger than that from the kaon channel. 
The transverse mass spectra in the kaonic channel with medium effects (solid triangles in Fig. 2) reveals nearly identical slope as for the bare masses. On the other hand, the large number of dimuon production at low $m_{T}$ in the early stage of collisions with in-medium masses leads to a slightly steeper mass spectra with a slope parameter of $T=220 \mathrm{MeV}$. This is clearly seen in the inset of Fig. 2 (triangles) for the ratio $R\left(m_{T}\right)$ where the suppression at low $m_{T}$ is $\simeq 33 \%$.

\subsection{RHIC}
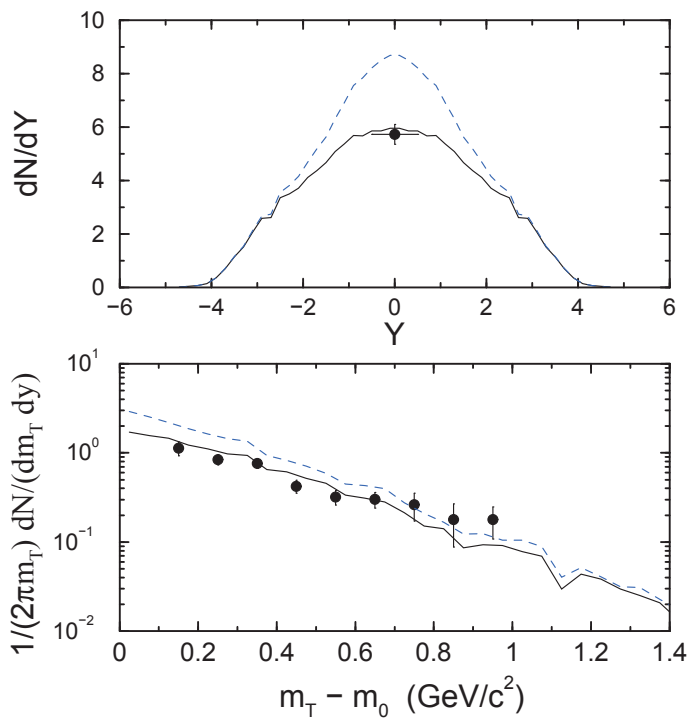

Fig. 3. The rapidity distribution (top panel) and the transverse mass spectra (bottom panel) for midrapidity $(|y|<$ $0.5)$ phi mesons reconstructed from $K^{+} K^{-}$pairs (solid curves) and from $\mu^{+} \mu^{-}$channel (dashed curves) for $\mathrm{Au}+\mathrm{Au}$ collisions at RHIC energy of $\sqrt{s}=130 \mathrm{~A} \mathrm{GeV}$ at an impact parameter of $b \leq 5.3 \mathrm{fm}$ in the AMPT model. The solid circles are the STAR experimental data [40] from $0-11 \%$ central collisions for $\phi$ reconstructed from $K^{+} K^{-}$ decay.

In the AMPT model, heavy ion collisions at RHIC differ from that at SPS mainly in the increasing effect of minijet gluons. However, the initial heavy ion collision dynamics at RHIC remains dominated by soft string fragmentation processes as at SPS. Fig. 3 (top panel) shows the results from the AMPT model for the rapidity distribution of phi mesons reconstructed from $K^{+} K^{-}$and $\mu^{+} \mu^{-}$pairs for central $\mathrm{Au}+\mathrm{Au}$ collisions at the RHIC energy of $\sqrt{s}=130 \mathrm{~A} \mathrm{GeV}$. Of the total phi meson yield of 37 per event, about $24 \%$ of these are lost in the kaonic channel by hadronic rescattering and absorption. In spite of enhanced phi meson production in the early stages of the collision as compared to the SPS energy, its abundance at $y \approx 0$ is only about a factor of 1.5 large in the dimuon channel. This may be attributed to fewer kaons lost by rescattering at RHIC as they can escape rapidly out of the collision zone unperturbed due to of their large energies. It is seen that phi meson multiplicities reconstructed from $K^{+} K^{-}$pairs at midrapidity in the AMPT model is consistent with the STAR data [40].

The transverse mass spectra of $\phi$ meson from the two channels are shown in the bottom panel of Fig. 3, and compared with the STAR data [40] for the $K^{+} K^{-}$channel. Compared to a slope parameter of $T=379 \pm 50 \mathrm{MeV}$ in the data, the AMPT model predicts a smaller value of $T=335 \mathrm{MeV}$ in the kaonic channel in the range $0<m_{T}-m_{\phi}<1 \mathrm{GeV}$. The slope parameter for phi mesons determined from the $\mu^{+} \mu^{-}$channel is $T=297 \mathrm{MeV}$ resulting in a suppression factor at low $m_{T}$ of $R\left(m_{T}\right)=58 \%$. 
At both SPS and RHIC energy, the slope parameter of phi mesons from $K^{+} K^{-}$decay is, however, smaller compared to the experimental data, and this is likely due to the fact that the initial strings are basically noninteracting in the default AMPT model used in this study. Using the string melting version of the AMPT model, which would lead to strong partonic scattering, is expected to give a better description of the phi meson transverse momentum spectra.

\section{Summary}

We have briefly reviewed the AMPT model but have given a relatively detailed description of the scattering processes in the hadronic phase. Using the phi meson as an example, we have discussed via the default AMPT model, which only has minijets during the initial partonic stage, how its yield depends on whether it is measured through its dikaon or dimuon decay channels. Considering all possible collisions of $\phi$ with the hadronic medium, we have found that the phi meson yield from dimuon channel is about a factor of 1.7 and 1.5 higher than the kaon-antikaon channel for central $\mathrm{Pb}+\mathrm{Pb}$ collisions at $E_{\mathrm{lab}}=158 \mathrm{~A} \mathrm{GeV}$ at SPS and $\mathrm{Au}+\mathrm{Au}$ collisions at $\sqrt{s_{N N}}=130 \mathrm{GeV}$, respectively. The larger phi meson yield in the dilepton than in the dikaon channel is because kaons originating from phi meson decay in the dense hadronic medium are predominantly scattered and absorbed and thus do not contribute to the phi meson yield, whereas all the $\mu^{+} \mu^{-}$pair freely escape. Also, we have demonstrated that medium modifications of the phi meson properties as well as those of kaons from its decay have significant effects on the phi meson yield and transverse momentum spectrum from the dilepton channel. Furthermore, we have compared results from the AMPT model with experimental data from heavy ion collisions at both SPS and RHIC. For central $\mathrm{Pb}+\mathrm{Pb}$ collisions at $158 \mathrm{~A} \mathrm{GeV}$ at SPS, our results agree with the $\phi \rightarrow K^{+} K^{-}$measurements by NA49 [24] but is about a factor of two smaller than the $\phi \rightarrow \mu^{+} \mu^{-}$measurements by NA50 [25]. For heavy ion collisions at RHIC energy of $\sqrt{s}=130 \mathrm{~A} \mathrm{GeV}$, the AMPT model gives the yield and slope parameter of phi mesons from the $K^{+} K^{-}$channel that are in reasonable agreement with the STAR data. More recent CERES data from $\mathrm{Pb}+\mathrm{Au}$ collisions [41] and NA 60 data from In-In collisions [42] at SPS as well as STAR data from $\mathrm{Au}+\mathrm{Au}$ collisions at RHIC $[43,44]$ indicate, however, similar yields of phi mesons from both the dikaon and dilepton channels. The difference between our results based on the AMPT model and these recent experimental observations could be due to the long hadronic phase in the default version of AMPT, which does not include the expected initial partonic phase except that of the minijets, used in this study. With the string melting version of the AMPT model, which includes an initial partonic stage consists of valence quarks and/or antiquarks from hadrons that would have been produced during initial collisions, the hadronic phase is shorter and also has a lower density than in the default version of AMPT. This would reduce dilepton emission from phi mesons and rescattering of kaons and antikaons from phi meson decay in the medium, thus reducing the difference between the yield and spectrum of phi mesons determined from the dilepon and dikaon channels.

We have not addressed the question of phi meson invariant mass spectrum from the dilepton and dikaon channels. Although the phi meson dikaon invariant mass spectrum is expected to be similar to that in vacuum as it essentially reflects only those kaons and antikaons which have not been rescattered in the medium, this is not the case for the dilepton invariant mass sepctrum. According to Ref. [45], including medium effects would lead to a broadening of dilepton invariance spectrum near phi meson peak due to the increase in its decay width as well as a shoulder structure below the bare phi meson mass as a result of the dropping phi meson in-medium mass. Also, it was shown in Ref. [46], the shoulder structure could turn into a lower mass peak in the dilepton invariant mass spectrum if there is a first-order or long crossover phase transition from the QGP to the hadronic matter in relativistic heavy ion collisions. The experimental measurement of the dilepton invariant mass spectrum near the phi meson mass from heavy-ion collisions is thus a useful tool for studying the phi meson properties in dense matter and the properties of the QCD phase transition. More generally, studying resonances production in relativistic heavy ion collisions provides the possibility to probe the properties of produced hot dense matter and its constituents, particularly the strongly interacting resonances. 


\section{References}

1. H. van Hees and R. Rapp, Phys. Rev. Lett. 97 (2006) 102301.

2. O. Linnyk, E. L. Bratkovskaya, V. Ozvenchuk, W. Cassing, and C. M. Ko, Phys. Rev. C 84 (2011) 054917.

3. B. I. Abelev et al. (STAR Collaboration), Phys. Rev. Lett. 97 (2006) 132301.

4. P. Braun-Munzinger, D. Magestro, and J. Stachel, Phys. Lett. B 518 (2001) 41.

5. M. Bleicher and J. Aichelin, Phys. Lett. B 530 (2002) 81.

6. Z. W. Lin, C. M. Ko, B. A. Li, B. Zhang, and S. Pal, Phys. Rev. C 72 (2005) 064901.

7. S. Pal, C. M. Ko, and Z. W. Lin, Nucl. Phys. A 707 (2002) 525.

8. M. Gyulassy and X. N. Wang, Comp. Phys. Comm. 83 (1994) 307.

9. B. Zhang, C. M. Ko, B. A. Li, Z. W. Lin, and B.H. Sa, Phys. Rev. C 62 (2000) 054905.

10. B. Zhang, Comp. Phys. Comm. 109 (1998) 193.

11. T. Sjöstrand, Comp. Phys. Comm. 82 (1994) 74.

12. B. A. Li and C. M. Ko, Phys. Rev. C 52 (1995) 2037.

13. Z. W. Lin and C. M. Ko, Phys. Rev. C 65, 034904 (2002).

14. S. Pal, C. M. Ko, and Z. W. Lin, Nucl. Phys. A 730 (2004) 143.

15. B. A. Li, and C. M. Ko, Nucl. Phys. A 646 (1999) 481

16. J. Cugnon, P. Deneye, and J. Vandermeulen, Phys. Rev. C 41 (1990) 1701.

17. G. E. Brown, C. M. Ko, Z. G. Wu, and L. H. Xia, Phys. Rev. C 43 (1991) 1881.

18. Z.W. Lin, C.M. Ko, and S. Pal, Phys. Rev. Lett. 89 (2002) 152301.

19. W. Liu, C. M. Ko, and L. W. Chen, Nucl. Phys. A 765 (2006) 401.

20. Z. W. Lin, S. Pal, C. M. Ko, B. A. Li, and B. Zhang, Phys. Rev. C 64 (2001) 011902(R).

21. W. S. Chung, G. Q. Li, and C. M. Ko, Nucl. Phys. A 625 (1997) 347.

22. C. M. Ko, and B. H. Sa, Phys. Lett. B 258 (1991) 6.

23. L. Alvarez-Ruso and V. Koch, Phys. Rev. C 65 (2002) 054901.

24. S. V. Afanansiev et al. (NA49 Collaboration), Phys. Lett. B 491 (2000) 59.

25. M. C. Abreu et al. (NA50 Collaboration), J. Phys. G 27 (2001) 405.

26. S. C. Johnson, B. V. Jacak, and A. Dress, Eur. Phys. J. C 18 (2001) 645.

27. J. Schaffner, J. Bondorf, and I. N. Mishustin, Nucl. Phys. A 625 (1997) 325.

28. G. E. Brown, C. H. Lee, M. Rho, and V. Thorsson, Nucl. Phys. A 567 (1994) 937.

29. C. M. Ko, and G.Q. Li, J. Phys. G 22 (1996) 1673; C.M. Ko, V. Koch, and G.Q. Li, Annu. Rev. Nucl. Part. Sci. 47 (1997) 505.

30. G. Q. Li, C. H. Lee, and G. E. Brown, Nucl. Phys. A 625 (1997) 372.

31. G. Q. Li and G. E. Brown, Nucl. Phys. A 636 (1998) 487.

32. S. Pal, C. M. Ko, Z. W. Lin, and B. Zhang, Phys. Rev. C 62 (2000) 061903(R).

33. T. Barnes and E. S. Swanson, Phys. Rev. C 49 (1994) 1166.

34. E. Friedman, A. Gal, and C. J. Batty, Phys. Lett. B 308 (1993) 6; Nucl. Phys. A 579 (1994) 518;

E. Friedman, A. Gal, J. Mares and A. Cieplý, Phys. Rev. C 60 (1999) 024314.

35. G. Q. Li, C. H. Lee, and G. E. Brown, Phys. Rev. Lett. 79 (1997) 5214.

36. T. Hatsuda and S. H. Lee, Phys. Rev. C 46 (1992) R34.

37. M. Asakawa and C. M. Ko, Nucl. Phys. A 572 (1994) 732.

38. F. Klingl, T. Waas, and W. Weise, Phys. Lett. B 431 (1998) 254.

39. V. Friese, Dissertation (1999), University of Marburg; D. Röhrich, J. Phys. G. 27 (2001) 355.

40. C. Adler et al., Phys. Rev. C 65 (2002) 041901(R).

41. D. Adamová et al. (CERES Collaboration), Phys. Rev. Lett. 96 (2006) 152301.

42. R. Arnaldi et al. (NA60 Collaboration), Eur. Phys. J. C 64 (2009) 260.

43. B. I. Abelev et al. (STAR Collaboration), Phys. Rev. C 79 (2009) 064903.

44. M. Wada for the STAR Collaboration in this proceedings.

45. W. S. Chung, C. M. Ko, and G. Q. Li, Nucl. Phys. A 641 (1998) 357.

46. M. Asakawa and C. M. Ko, Phys. Lett. B 322 (1994) 33; Phys. Rev. C 50 (1994) 3064; Nucl. Phys. A 566 (1994) 447c. 